\title{
Die Datierung der Nomarchen von Hermopolis aus dem frühen Mittleren Reich - eine Phantomdebatte?*
}

\section{Vorbemerkungen}

Einmal mehr wird es in diesem Beitrag um die Datierung einiger Graffiti gehen, die Nomarchen („Gaufürsten“) aus Hermopolis bzw. die von ihnen gesandten Beamten im Steinbruch von Hatnūb hinterlassen haben. Nachdem diese Problematik bereits hinlänglich diskutiert wurde und nach einer sehr kontrovers, mit Vehemenz und großer Nachdrücklichkeit geführten wissenschaftlichen Debatte in den 60er bis $80 \mathrm{er}$ Jahren inzwischen als weitestgehend gelöst angesehen werden kann, sollte zu weiteren Anmerkungen eigentlich kein Anlass bestehen: Es scheint alles gesagt zu sein. Aus diesem Grund sei vorausgeschickt, dass es in den folgenden Ausführungen nicht um einen neuen zeitlichen Ansatz für die Graffiti von Hatnūb, insbesondere die aus der Zeit des Nhr.i, geht - und der letztlich nur eine überholte Ansicht wiedergeben könnte. Es soll vielmehr gezeigt werden, dass der Zugang zu dieser Frage wesentlich einfacher sein könnte, als es bislang gesehen wurde. Es soll also ein anderer Weg begangen werden, mit dem sich die Texte ebenfalls zeitlich einordnen lassen und dies zum Teil sogar noch genauer als bisher.

Es sind mehrere Quellengruppen, die einen Zugang zur Situation des 15. oberägyptischen Nomos im frühen Mittleren Reich erlauben. Während das weltliche Zentrum in Hermopolis, das alte $W n . w$ und Hmn.w und heutige al-Ašmūnain, seine archäologische Hinterlassenschaft nur zögerlich freigibt, lassen sich zahlreiche

* Danken möchte ich Burkhard Backes und Wolfgang Schenkel für die Lektüre eines ersten Entwurfs dieses Beitrags, ihre wohlwollende Kritik sowie ihre Hinweise und Anregungen.

${ }^{1} \mathrm{Zu}$ Siedlungsschichten aus dem Mittleren Reich D. Kessler, Historische Topographie der Region zwischen Mallawi und Samalut, TAVO Reihe B, Nr. 30, Wiesbaden 1981, S. 83 ff. mit weiteren Hinweisen.
Informationen in den Gräbern der zugehörigen Nekropole in Dair al-biršā auf dem Ostufer des Nils entnehmen, weitere den Graffiti im großen Steinbruch von Hatnūb (dazu jeweils noch im Folgenden). Letzterer gehört, ebenso wie ein kleinerer, jüngerer Steinbruch, an sich schon zum Gebiet des 14. oberägyptischen Nomos, ist aber über die Zugangswege dem Nomos von Wn.t faktisch und verwaltungstechnisch angegliedert gewesen ${ }^{2}$. Die Informationen, die diese Quellen vermitteln, sind von teilweise sehr unterschiedlicher Art. Sie geben Auskunft über Grabarchitektur, ihre Entwicklung sowie lokale Ausprägung und über die Dekoration der Gräber, über Totenliteratur und speziell Sargtexte sowie Grabinventar, Restauration, über Expeditionen und Expeditionswesen. Zugleich lassen sich personelle Überschneidungen erkennen, so dass Verknüpfungen zwischen den Quellengruppen möglich sind. Es erschließt sich so ein Gebiet auf eine Weise, die andernorts nur selten gegeben ist ${ }^{3}$. Dieser Vorteil ist allerdings zunächst nur eingeschränkt zu nutzen gewesen, da die zeitliche Einordnung lange als umstritten bzw. unsicher gelten musste. Allein die Datierung „frühes Mittleres Reich“ konnte als durchgängig akzeptiert gelten. Die Unsicherheit hängt damit zusammen, dass keine der (frühen) Quellen Königsnamen nennt oder sogar Regierungsjahre eines Königs. Dies ist erst mit Sesostris I. der Fall, aus dessen Regierungszeit uns einige Graffiti in Hatnūb überliefert sind.

Einige Restaurationsinschriften in Gräbern von aš-Šaih Sa ${ }^{\leftarrow}$ īd, der südlich von Dair al-biršā gelegenen Nekropole des Alten Reiches, sind gleichfalls von Interesse, können hier aber unberücksichtigt bleiben.

${ }^{3}$ Vergleichbar ist vielleicht die Situation im 1. oberägyptischen Nomos mit den Quellen von Elephantine, Qubbat al-Hawā' und Sehel. 


\section{Wissenschaftsgeschichtliche Vorgaben}

Die Diskussion um die Datierung der Graffiti von Hatnūb geht auf das Jahr 1891 zurück$^{4}$, als während eines Surveys des EEF in Dair al-biršā auch dem größeren der beiden Steinbrüche von Hatnūb ein Besuch abgestattet wurde. In ihrer Publikation zu den Gräbern von Dair al-biršā haben F. Ll. Griffith und P. E. Newberry insgesamt sieben dieser Graffiti wiedergegeben und auch hinsichtlich ihrer Aussagekraft ausführlich besprochen und bewertet ${ }^{5}$. Diese Überlegungen machten erstmals die Bedeutung der Inschriften erkennbar und gaben eine Ahnung davon, welch wichtige Angaben sie enthalten und welche Bereicherung sie darstellen, insbesondere mit Blick auf die Gräber von Dair al-biršā. Die erste gröBere Dokumentation in beiden Steinbrüchen erfolgte 1892 durch M. W. Blackden und G. W. Fraser, die allerdings infolge ihres internen Charakters keine weite Verbreitung fand ${ }^{6}$. Sie wurde aber die Grundlage für die Arbeiten von G. Möller, der während eines Aufenthaltes 1907 sämtliche Inschriften in den beiden Steinbrüchen wissenschaftlich dokumentierte, aber auch die Situation im Gelände u. a.m. festhielt ${ }^{7}$. Es blieb G. Möller auf Grund des historischen Zeitgeschehens und in Folge seines frühen Todes 1921 versagt, diese umfassende und grundlegende Dokumentation auch zu bearbeiten und für eine Veröffentlichung vorzubereiten. Diese Aufgabe fiel R. Anthes zu. Er legte 1928 die Publikation der Texte aus Hatnūb vor, die sich

Zur Forschungsgeschichte R. Anthes, Die Felseninschriften von Hatnub nach den Aufnahmen Georg Möllers, UGAÄ IX, Leipzig 1928, S. 1 ff.

F. Ll. Griffith/P. E. Newberry, El Bersheh II, ASE 4, London 1895, Tfn. XXIIf., zur Diskussion der Aussagen in den Graffiti op. cit., S. 1-16 und S. 47-54; s. a. W. M. F. Petrie, Tell el Amarna, London 1894, Tf. XLII, mit der Wiedergabe einiger kleinerer Inschriften, die er 1892 aufgenommen hatte.

Vgl. hierzu die Angaben von Anthes, Hatnub (Anm. 4), S. 1. Weder A. Kamal und G. Daressy, die 1897-1902 in Dair al-biršā arbeiteten, noch G. Reisner, der dort 1915 eine Unternehmung führte, scheinen die Steinbrüche von Hatnūb besucht zu haben.

G. Möller, Bericht über die Aufnahme der hieroglyphischen und hieratischen Felsinschriften im Alabasterbruch von Hatnub in Mittelägypten, SPAW 1908, S. 679-690. auf das Material stützte, das bereits G. Möller gesammelt hatte, aber auch einige neuere Angaben und Aufnahmen mit einbezog ${ }^{8}$. Abgesehen von Dokumentation und philologischer Bearbeitung der Inschriften brachte R. Anthes die Texte in eine relative (zeitliche) Ordnung, die nach wie vor weitgehende Gültigkeit besitzt. Die Publikation von Anthes legte zugleich den Grundstein für ein erstes Verständnis der Texte und damit für eine Diskussion, die zeitweilig mit großer Lebhaftigkeit geführt wurde. In dieser Diskussion ging es um die Datierung der Graffiti, zugleich aber um nicht weniger als das Bild einer ganzen Epoche, der sogenannten 1. Zwischenzeit und des frühen Mittleren Reiches. Im Mittelpunkt der Überlegungen von R. Anthes standen die Graffiti aus der Zeit des Nhr.i, sie wurden zum Kernpunkt auch für jede weitere Diskussion um die zeitliche Einordnung der Texte. Das Besondere dieser Graffiti sind die Anspielungen, dann aber auch sehr ausführlichen Schilderungen kämpferischer Auseinandersetzungen, Unruhen und Hungersnot im Land, die sie beinhalten und die sich nur als Bürgerkriegshandlungen und -zustände interpretieren lassen. Die in den Graffiti beschriebenen Kämp$\mathrm{fe}$, an denen in unterschiedlicher Weise Nhr.i selbst wie auch seine beiden Söhne beteiligt waren, brachte R. Anthes mit den militärischen Auseinandersetzungen in Verbindung, die in Zusammenhang mit der neuerlichen Reichseinigung und der Gründung des Mittleren Reiches unter Mentuhotep II. stattgefunden haben, und er datierte sie in die ausgehende 1. Zwischenzeit bzw. in vorthebanische Zeit ${ }^{9}$. Indizien für diesen frühen zeitlichen Ansatz sah R. Anthes in der Titelverteilung bei Nhr.i und dem Vergleich einzelner Titel mit solchen, die in den Gräbern von Asyūt belegt sind. Entsprechend ging er von einem nur kurzen zeitlichen Abstand zwischen den in Asyūt geschilderten Ereignissen aus dem Ende der Herakleopolitenzeit und den Schilderungen von Nhr.i aus. Die aus den Titeln des

Anthes, Hatnub (Anm. 4).

Ausführlich dargelegt Anthes, Hatnub (Anm. 4), S. 93 ff., s. aber schon id., Die zeitliche Ansetzung des Fürsten Nhrj I. vom Hasengau, in: ZÄS 59, 1924, S. $100-108$. 
Nhr.i abzuleitende Vorrangstellung gegenüber dem König sei - so R. Anthes - in den Kämpfen begründet, die in den Graffiti beschrieben sind und an denen Nhr.i an der Seite des herakleopolitanischen Königs gegen die vordrängende 11. Dynastie teilgenommen habe. Dass es sich um gerade diese Parteinahme handelt, ergebe sich aus der Lage von Asyūṭ. Diese von R. Anthes vorgenommene zeitliche und inhaltliche Einordnung von Nhr.i blieb lange Zeit unwidersprochen, wenngleich verschiedentlich Modifizierungen und abweichende Darstellungen hinsichtlich der Bündnisgenossen und der Parteilichkeit von Nhr.i geäußert wurden ${ }^{10}$. Auch das Bekanntwerden neuer Graffiti hatte auf die Einschätzungen von R. Anthes keinen Einfluss, da es sich dabei um spätere Texte handelt (zu diesen noch im Folgenden).

Erst mit den Ausführungen von W. Schenkel 1962 kam Bewegung in die Frage, wie die Graffiti des Nhr.i zu datieren sind ${ }^{11}$. Aufgrund paläographischer Beobachtungen sprach sich W. Schenkel für eine Einordnung der Graffiti in die Zeit von Amenemhet I./Sesostris I. aus. Die in Anbetracht dieser neuen Ausgangssituation notwendige Revidierung des bisherigen Datierungsansatzes veranlasste ihn zu der Annahme, dass die in den Texten angesprochenen Auseinandersetzungen auf Geschehnisse im Zuge des Regierungswechsels von Amenemhet I. zu Sesostris I. zurückgehen, und er schlug eine Datierung in das 6. bis (mindestens) 14. Jahr von Sesostris I. vor.

Diese fundamental abweichende Einschätzung der Texte blieb nicht lange unangefochten. Zunächst war es E. Blumenthal, die sich mit den Graffiti von Nhr.i auseinandersetzte und die Argumente für die bis dato vorgetragenen Meinungen vortrug und gegeneinanderstellte ${ }^{12}$. Ausführlich diskutierte sie die unterschiedlichen

S. E. Blumenthal, Die Datierung der NhriGraffiti von Hatnub. Zur Stellung der ägyptischen Gaufürsten im frühen Mittleren Reich, in: Altorientalische Forschungen IV, 1976, S. 35-62 (S. 35f.).

W. Schenkel, Frühmittelägyptische Studien, Bonner Orientalische Studien N. S. 13, Bonn 1962, S. $84-95$.

${ }^{12}$ Blumenthal, in: Altorientalische Forschungen IV, 1976 (Anm. 10), S. $35 \mathrm{ff}$.
Einschätzungen zur Genealogie und Chronologie, Paläographie, Archäologie sowie Phraseologie und lieferte mit den von ihr in dem Artikel gleichfalls zusammengestellten Überlegungen einen wichtigen Beitrag zur Datierung der Graffiti. In einer abschließenden Beurteilung sprach sie sich zwar gegen den von W. Schenkel vorgeschlagenen späten Ansatz aus und für die Datierung von R. Anthes, blieb bei einer genaueren zeitlichen Einordnung des Nhr.i letztlich aber vage. Anders E. Brovarski ${ }^{13}$. Mit seiner Bearbeitung einiger Särge aus Dair al-biršā und seinen Ausführungen zu Paläographie, Epigraphik, Phraseologie und Ikonographie insbesondere der Gräber und Inschriften von ' $h 3-n h t$ (I.) und $N h r . i$ (I.) brachte er weitere wichtige Impulse in die Diskussion ein und äußerte sich zugleich dahingehend, dass die frühesten aus Dair albiršā bekannt gewordenen Särge B1-4Bo und $\mathrm{B} 8 \mathrm{Bo}$ vornehmlich aus stilistischen Gründen in die 11. Dynastie gehörten und keinesfalls später zu datieren seien ${ }^{14}$.

Es war schließlich H. Willems, der mit seinen Untersuchungen zu Beginn der 80er Jahre den Lösungsansatz fand, der inzwischen als allgemein akzeptiert gelten $\mathrm{kann}^{15}$. H. Willems teilte die grundsätzliche Kritik von W. Schenkel an einer zu frühen Datierung der Graffiti des Nhr.i, übernahm aber nicht dessen späten Ansatz, sondern sprach sich für eine Datierung der Graffiti in den Beginn der 12. Dynastie aus. In den Inschriften aus der Zeit des Nhr.i sind nach seiner Auffassung Auseinandersetzungen ange-

${ }^{13}$ E. Brovarski, Ahanakht of Bersheh and the Hare Nome in the First Intermediate Period and Middle Kingdom, in: W. K. Simpson/W. M. Davis (Hrg.), Studies in Ancient Egypt, the Aegean, and the Sudan. Essays in Honor of Dows Dunham on the Occasion of his 90th Birthday, June 1, 1980, Boston 1981, S. 14-30.

Brovarski, in: Fs Dunham (Anm. 13), S. 28-30. Auch W. K. Simpson, Hatnub, in: LÄ II, Wiesbaden 1977, Sp. 1043-1045, und F. von Känel, Les prêtres ouâb de Sekhmet et les conjurateurs de Serket, Paris 1984, S. 17, sprechen sich für eine frühe Datierung in die 11. Dynastie aus, s. a. S. Giuliani, A New Proposal for the Interpretation of Hatnub Graffito $\mathrm{N}^{\circ} 16$, in: GM 159, 1997, S. 53-60 (S. 53 f.), die gleichfalls explizit den Datierungsansatz von R. Anthes zugrundelegt.

${ }^{15} \mathrm{H}$. Willems, The Nomarch of the Hare Nome and Early Middle Kingdom History, in: JEOL 28, 1983-1984, S. 80-102. 
sprochen, zu denen es in Zusammenhang mit der Machtübernahme von Amenemhet I. kam. Grundlage für diese Einschätzung sind neben Überlegungen zur Genealogie vor allem Untersuchungen zur Paläographie der Inschriften und Phraseologie der Texte sowie zur Typologie und Stilistik der Särge aus Dair al-biršǎa ${ }^{16}$. Dieser zwischen den beiden früheren, ausgesprochen konträren Datierungen angesiedelte Ansatz hat mittlerweile allgemeine Zustimmung gefunden und ist zusätzlich untermauert worden ${ }^{17}$. Von $\mathrm{H}$. Willems selbst wurde er der archäologischen Unternehmung in Dair al-biršā zugrunde gelegt ${ }^{18}$.

\section{Die relative Chronologie der Nomarchen}

Es sind seit der (wissenschaftlichen) Entdeckung der Graffiti von Hatnūb und der Gräber von Dair al-biršā sehr unterschiedliche Kriterien gewesen, mit denen eine Datierung der jeweiligen Quellen versucht wurde. Dies dürfte trotz der Kürze bei der Darstellung der wissenschaftsgeschichtlichen Vorgaben bereits deutlich geworden sein. Von Beginn an wurden die genealogischen Beziehungen sehr stark berücksichtigt. Mit einbezogen wurden aber auch archäologische Kriterien, ferner Phraseologie und Paläographie der Texte. Was auf dieser Grundlage erarbeitet und - nicht zuletzt wegen der Eindeutigkeit der Quellen in diesem Punkt schon früh zu einem allgemeinen Konsens ge-

${ }^{16}$ Dazu Willems, in: JEOL 28, 1983-1984 (Anm. 15), S. 86 ff.; s. a. id., Chests of Life. A Study of the Typology and Conceptual Development of Middle Kingdom Standard Class Coffins, MVEOL XXV, Leiden 1988, S. $70 \mathrm{ff}$.

Vgl. etwa die Untersuchungen von G. Lapp, Typologie der Särge und Sargkammern von der 6. bis 13. Dynastie, SAGA 7, Heidelberg 1993, S. $71 \mathrm{ff}$. und S. $274 \mathrm{ff}$., zur Diskussion der bisherigen Datierungsansätze op. cit., S. $86 \mathrm{ff}$.

${ }^{18}$ E. Brovarski et al., Bersheh Reports I. Report of the 1990 Field Season of the Joint Expedition of the Museum of Fine Arts, Boston, University Museum, University of Pennsylvania, Leiden University, Boston 1992; H. Willems et al., Deir al Barsha. Volume 1: The Rock Tombs of Djehutinakht (No. 17L20/1), Khnumnakht (17L20/2), and Iha (17L20/3), With a Chapter on the History and Functioning of Nomarchal Rule in the Early Middle Kingdom (angekündigt). bracht werden konnte, war die relative Chronologie der Nomarchen bzw. ihrer Familien.

Insgesamt vier Nomarchen sind aus Inschriften in Hatnūb bekannt: ' $h 3-n h t$ (I.), Nhr.i (I.) sowie Dhw.ti-nht (IV.) und Dhww.ti-nht (V.) (zu Imn.w-m- $h$ 3.t $\mathrm{s}$. im Folgenden). Bei einigen von ihnen wissen wir, wo ihre Gräber in Dair al-biršā liegen, und wir kennen aus den Familien dieser Männer - aus Dair al-biršā und/oder Hatnūb weitere Mitglieder, von denen einige ebenfalls Nomarchen waren. Danach lässt sich die Abfolge (bis auf zwei Fälle) sicher rekonstruieren ${ }^{19}$.

(1) Als erster Nomarch des Mittleren Reiches amtierte (h3-nht (I.), aus dessen Amtszeit sich Gr. 12 (mit einer Datierung „13. Jahr") und Gr. 10 (,20. Jahr") erhalten haben. Gr. 11 („30. Jahr") kann ihm nicht sicher zugewiesen werden, weil an entscheidender Stelle zerstört.

(2) und (3) Dhhw.ti-nht (IV.), einer der Söhne von `h3-nht (I.), hat ein Graffito hinterlassen (Gr. 42), das allerdings den Nomarchentitel nicht nennt, und auch Gr. 11 (s. zuvor) muss eventuell ihm zugeschrieben werden. Vermutlich ist auch 'h3-nht (II.), ein weiterer Sohn, seinem Vater im Nomarchenamt nachgefolgt $t^{20}$. Für diese Annahme hat sich inzwischen ein weiteres Indiz ergeben, nachdem 1988 direkt westlich neben Grab 5 von 'h3-nht (I.) ein bis dato unbekanntes Grab entdeckt wurde (Grab X). Es gehört einem weiteren Nomarchen, bei dem es sich nach den auf einem Block erhaltenen Resten um einen zweiten ${ }^{\Upsilon h} 3$-nht handeln könnte ${ }^{21}$.

(4) Aus der Amtszeit von Nhr.i (I.) stammen mehrere Graffiti, die in das 4. (Gr. 14/15), 5. (Gr. 16-19), 6. (Gr. 20/21), 7. (Gr. 22-25) und 8. Jahr datieren (Gr. 26-28). Angebracht wurden sie von seinen beiden Söhnen oder von Personen, die zu seiner Beamtenschaft gehören.

19 Zur relativen Abfolge s. a. Brovarski, in: Fs Dunham (Anm. 13), S. 22f., Willems, in: JEOL 28, 1983-1984 (Anm. 15), S. 82f. und S. 102; id., Chests of Life (Anm. 16), S. 71, zu den angegebenen Graffiti vgl. Anthes, Hatnub (Anm. 4).

${ }^{20}$ Auch Willems, in: JEOL 28, 1983-1984 (Anm. 15), S. 82, sieht in beiden Söhnen von `h3-nht (I.) Nachfolger im Amt.

${ }^{21}$ Bersheh Reports I (Anm. 18), S. 41; H. Willems, Deir el-Bersheh. Preliminary Report, in: GM 110, 1989, S. 75-95 (S. 79 ff. und Plan S. 91). 
Für Nhr.i (I.) wurde Grab EEF 4/R 19 in Dair al-biršā angelegt .

(5) Unter Dhw.ti-nht (V.), einem Sohn von Nhr.i (I. $)^{23}$, wurde Gr. 31 (,2. Jahr ${ }^{\circ}$ ) angebracht. Leider weist die Inschrift eine Reihe von Zerstörungen auf, die besonders bedauerlich sind, da der Text offensichtlich von den Zeiten (zumindest) zweier verschiedener Nomarchen spricht. Auch Gr. 32 („20. Jahr") kann inzwischen diesem Dhw.ti-nht zugewiesen werden, nachdem die ursprüngliche Lesung des Personennamens Dhw.ti-htp durch G. Möller und auch R. Anthes von E. Brovarski korrigiert werden konnte ${ }^{24}$.

(6) Nachfolger von Dhw.ti-nht (V.) wurde Nhr.i (II.), vermutlich ein Neffe von Dhww.ti-nht, Sohn von dessen Bruder K3y ${ }^{25}$. Dass Nhr.i (II.) als Nomarch tätig war, ergibt sich zum einen aus sehr viel späterer Zeit, und zwar aus dem Grab seines Enkels Dhw.ti-htp, ebenfalls Nomarch. Dort ist Dhw.ti-htp als Nachfolger eines Nhr.i, in dem man $N h r . i$ (II.) erkennen darf, dargestellt ${ }^{26}$. Zudem ist der Titel eines Nomarchen nun auch im Grab EEF 7/R 1 (B1B) eines Nhr.i belegt, das $N h r . i$ (II.) gehören dürfte ${ }^{27}$.

(7) und (8) Von Nhr.i (II.) wissen wir, dass er zumindest einen Sohn hatte, 'Imn.w-m-h3.t. Dieser ist Besitzer von Grab EEF 3/R 18 in Dair albirša ${ }^{28}$ und hat in Hatnūb ein Graffito (Gr. 49) mit der Datierung „31. Jahr Sesostris’ I.“ hinterlassen. Das Graffito nennt für 'Imn.w-m-h3.t zwar den höchsten Rangtitel, bezeichnet ihn aber nicht als Nomarchen. Er hat diese Funktion also möglicherweise erst später, nach diesem Zeit-

${ }^{22}$ Bersheh Reports I (Anm. 18), S. $28 \mathrm{ff}$.

${ }^{23}$ Zur Familie des Nhr.i Willems, in: JEOL 28, 1983-1984 (Anm. 15), S. 82.

${ }^{24}$ Brovarski, in: Fs Dunham (Anm. 13), S. 27, Anm. 107; s. a. Willems, in: JEOL 28, 1983-1984 (Anm. 15), S. 82.

${ }^{25} \mathrm{Zu}$ den Zusammenhängen im einzelnen s. die Ausführungen von Willems, op. cit., S. 82 f.

${ }^{26}$ P. E. Newberry, El Bersheh I, ASE 3, London 1895, Tf. XXXIII; dazu auch Willems, in: JEOL 28, 1983-1984 (Anm. 15), S. 83.

${ }^{27}$ Schon Anthes, Hatnub (Anm. 4), S. 100, spricht sich für diese Identifizierung aus, s. a. Griffith/ Newberry, El Bersheh II (Anm. 5), S. 37 und Tfn. XVIIIf., Brovarski, in: Fs Dunham (Anm. 13), S. 22. Zu Grab EEF 7/R 1 Bersheh Reports I (Anm. 18), S. 13-27.

${ }_{28}$ Bersheh Reports I (Anm. 18), S. $27 \mathrm{f}$. punkt, ausgeübt. Die Namensgleichheit der Eltern von 'Imn.w-m-h3.t mit denen von Dhw.ti-nht (VI.), Besitzer des stark zerstörten Grabes EEF 1/R $21^{29}$ und ebenfalls Nomarch, macht die beiden sehr wahrscheinlich zu Brüdern ${ }^{30}$. Leider ist nicht festzulegen, in welcher Reihenfolge 'Imn.w-m-h3.t und Dhw.ti-nht (VI.) tätig waren. Tendenziell wird Dhw.ti-nht (VI.) als früherer der beiden Nomarchen angesehen ${ }^{31}$.

(9) Den Nomarchen Dhw.ti-htp kennen wir nur von dem Grab, das er sich in Dair al-biršā anlegen ließ (Grab 2), in den Texten von Hatnūb taucht er nicht auf. Wie schon erwähnt, handelt es sich bei ihm um den Nachkommen eines weiteren Sohnes von Nhr.i (II.) ${ }^{32}$.

(10) Als letzter Nomarch des Mittleren Reiches in Dair al-biršā muss wahrscheinlich Dhw.ti-nht VII. gelten, der bis in die Regierungszeit von Amenemhet III. amtiert haben dürfte ${ }^{33}$. Von diesem Nomarchen ist sein Sarg bekannt, der von einer Frau namens ' $n$ h.w usurpiert wurde (B14C), ferner sein Grab (Reisner 4/EEF $6)^{34}$.

Zeitlich vor diese Familien gehören die Nomarchen Dhw.ti-nht, Sohn des Dhw.ti-nht (Inschr. IX), und Dhw.ti-nht, Sohn des Hww (Inschr. X) ${ }^{35}$, die aber letztlich nur der Vollständigkeit halber hier erwähnt sind. Sie lebten vermutlich in der 9./10. Dynastie, was mit den erhaltenen Resten eines Königsnamens in beiden

${ }^{29}$ Bersheh Reports I (Anm. 18), S. 30-38.

${ }^{30}$ So schon Anthes, Hatnub (Anm. 4), S. 99 f.; s. a. Brovarski, in: Fs Dunham (Anm. 13), S. 23, Willems, in: JEOL 28, 1983-1984 (Anm. 15), S. 82f.; id., Chests of Life (Anm. 16), S. 74.

${ }^{31}$ Vgl. Brovarski, in: Fs Dunham (Anm. 13), S. 23; Bersheh Reports I (Anm. 18), S. 28.

${ }^{32}$ Willems, in: JEOL 28, 1983-1984 (Anm. 15), S. 83 .

${ }^{33}$ D. Bidoli, Die Sprüche der Fangnetze in den altägyptischen Sargtexten, ADAIK 9, Glückstadt 1976, S. 22, hatte mit einem Dhw.ti-nht VII., der zwischen Imn.w-m-h3.t und Dhw.ti-htp amtierte, noch Sarg B15C in Verbindung bringen wollen, der ist nun aber Dhw.ti-nht VI. zuzuweisen, s. zuvor.

${ }^{34} \mathrm{Zu}$ diesem Nomarchen Brovarski, in: Fs Dunham (Anm. 13), S. 23 und S. 25 f.; Willems, Chests of Life (Anm. 16), S. 79.

${ }^{35}$ Anthes, Hatnub (Anm. 4), S. 14-16 und Tfn. 6 f., des weiteren op. cit., S. 103. 
Graffiti in Einklang zu bringen ist ${ }^{36}$. Zusätzlich zu berücksichtigen ist ein Nomarch Th3 (Gr. 9). Dessen zeitliche Einordnung ist letztlich nicht zufriedenstellend möglich. Das Graffito trägt die Datierung 31. Jahr, zweiter Monat der 3h.t-Jahreszeit, muss also in der mindestens so langen Regierungszeit eines Herrschers entstanden sein. Dafür kämen nach den derzeit bekannten Regierungslängen Mentuhotep II. und Phiops II. in Frage. Für letzteren spricht vor allem die Anbringung der Inschrift inmitten von Graffiti des Alten Reiches ${ }^{37}$.

\section{Die Jahresangaben der Nomarchen}

Bis zum 22. Jahr von Sesostris I. (dazu noch im Folgenden) fehlen in den Graffiti von Hatnūb Datierungen, mit denen Regierungsjahr und Name des jeweils herrschenden Königs angegeben wären. Was aber in einer ganzen Reihe von Inschriften anzutreffen ist, das sind Jahresangaben ohne Königsnennung. Sie sind, aufgrund der ihnen auferlegten Interpretation, $\mathrm{zu}$ einem Charakteristikum der Graffiti von Hatnūb geworden, und auf sie soll im Folgenden näher eingegangen werden, da sie m. E. die maßgebliche Grundlage für eine absolute Datierung der Graffiti darstellen können.

Spätestens seit der Publikation von R. Anthes werden diese Jahresangaben, die jeweils am Anfang eines Graffitos stehen, auf den Nomarchen bezogen, dessen Name direkt dahinter steht, und es wird das angegebene Jahr als Datierung

${ }^{36} \mathrm{Zu}$ Inschr. X Schenkel, Frühmittelägyptische Studien (Anm. 11), S. 27 f.; Brovarski, in: Fs Dunham (Anm. 13), S. 26 f. mit Anm. 103; Willems, in: JEOL 28, 1983-1984 (Anm. 15), S. 87, zu den Königsnennungen in Inschr. IX und X J. von Beckerath, Die Dynastie der Herakleopoliten (9./10. Dynastie), in: ZÄS 93, 1966, S. 13-20 (S. 16).

${ }^{3}$ In diesem Sinn schon Anthes, Hatnub (Anm. 4), S. 23 mit Anm. 1, ähnlich, aber doch relativierend op. cit., S. 103 („Gewiß ganz nahe an die Zeit des A.R. führt uns das Graffito (9) eines $T h 3$ in Hatnub"). Brovarski, in: Fs Dunham (Anm. 13), S. 22, macht allerdings darauf aufmerksam, dass auf Grund der Paläographie das Graffito später als Phiops II. entstanden sein muss. nach eigenen Amtsjahren aufgefasst ${ }^{38}$. R. Anthes hat nicht ausgeführt, worin sich seine Annahme begründet, da sie sich nahtlos zu den Inhalten insbesondere der Graffiti des Nhr.i, zu dessen Zuständigkeit wie auch Selbstdarstellung, fügte. Danach habe Nhr.i auf Grund seiner auch militärischen Unterstützung für Pharao eine Ausnahmestellung eingenommen und selbstbewusst sein Amt ausgeübt, was auch die Datierung nach eigenen Amtsjahren und die Inanspruchnahme königlicher Privilegien z. B. in Form bestimmter Epitheta umfasste ${ }^{39}$. So findet sich in den Graffiti (nicht nur des Nhr.i ) z. B. die Verwendung

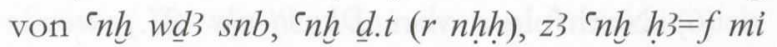
$R^{\top}(w)$ d.t oder $m 3^{\top} h r w$, oder es wurde ein Schwur auf den Namen des Nomarchen abgelegt, die göttliche Abkunft betont, des weiteren die Beschreibung der Expeditionen an die Phraseologie königlicher Inschriften angelehnt. Mit diesen Eigenheiten, so ist unterstellt worden, übernahmen die Beamten - und allen voran wieder Nhr.i (I.) - königliche Privilegien und bezogen sie auf ihre eigene Person.

Die Annahme, es handele sich bei den Jahresangaben um die Regierungsjahre der Nomarchen, ist augenscheinlich auch später nie hinterfragt worden ${ }^{40}$, wenngleich sich allmählich abweichende Datierungen für die Graffiti durchsetzten und sich auch die Einschätzungen hinsichtlich der Inhalte der Graffiti veränderten. Die Argumente für die privilegierte Stellung, mit der die Nomarchen vermeintlich in Konkurrenz zum Königshaus standen, sind aber nach und nach aufgehoben worden bzw. stehen der Tatsache gegenüber, dass die Nomarchen fest in die ägyptische Beamtenhierarchie eingebunden waren und ihre Arbeiten in Hatnūb im königlichen Auftrag durchgeführt wurden (dazu noch im Folgenden). Zudem hat die Auseinandersetzung

${ }^{38}$ Anthes, Hatnub (Anm. 4), S. 85 f., S. 98 und passim. So aber auch schon Griffith/Newberry, El Bersheh II (Anm. 5), S. 5.

${ }^{39}$ Ausführlich dargelegt bei Anthes, Hatnub (Anm. 4), S. $85 \mathrm{ff} ;$; Blumenthal, in: Altorientalische Forschungen IV, 1976 (Anm. 10), S. $37 \mathrm{ff}$.

${ }^{40}$ Ausdrücklich als die Angabe von Amtsjahren der Nomarchen auch bei Willems, in: JEOL 28, $1983-$ 1984 (Anm. 15), S. 84ff., aufgefasst. 
mit den vermeintlichen Besonderheiten Parallelen an anderen Orten zu Tage gefördert.

Die besondere Situation in Hatnūb, als solche durch die Inhalte der Texte des Nhr.i bzw. aus der Zeit des $N h r . i$ geprägt, hat also eine den üblichen Konventionen entsprechende Lesart der Jahresangaben verhindert. Im Gegenteil wurde in Verbindung mit dem propagierten Selbstbewusstsein ${ }^{41}$ der Nomarchen die Datierung nach deren Amtszeiten als ein wichtiger Baustein für dieses Bild benutzt. M. E. zu Unrecht - oder anders ausgedrückt: Selbst in der Wüste konnte ein ägyptischer Beamter nicht machen, was er wollte. In der Folge möchte ich in den Jahresangaben der Graffiti die Nennung eines königlichen Regierungsjahres erkennen, selbst wenn der jeweils herrschende Pharao nicht direkt erwähnt wird. Für diese Annahme spricht m. E. (1) die allgemeine Einschätzung, dass eine Datierung nach den eigenen Amtsjahren vor dem Gesamteindruck der Inhalte und Aussagen der Texte schlichtweg unhaltbar ist, und - zumindest in einigen Fällen - (2) die formale Anordnung der einzelnen Elemente innerhalb eines Graffitos.

(1) Wenngleich der Stolz und das Selbstbewusstsein über die eigene Stellung und die vollbrachten militärischen und sozialen Leistungen in den Graffiti aus der Zeit des Nhr.i nicht zu übersehen sind, so ist zugleich auch seine Einbindung in eine feste Hierarchie offensichtlich. Dazu sei auf Gr. 14 (,4. Jahr") verwiesen, das in der Amtszeit des Nhr.i von dem Vorsteher der Flotte Ntr.w-htp.w angebracht wurde. Für diesen sind nach den Aussagen des Textes zwei Instanzen relevant, zum einen sein „Herr“, von dem er gelobt wird (hzj.y $n(. i) n b=f$, Z. 3) und für den er seinen Dienst verrichtet (. . r ir.t sšm $n n b=f$, Z. 7), zum anderen das Königshaus. Dessen qnb.t jubelt wegen der besonderen Beliebtheit seines Herrn in der d3d3.t (?) (qnb.t n.t pr(.w)-nzw

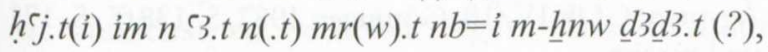
Z. 8f.). Zudem verrichtet Ntr.w-htp.w zwar Dienst für seinen Herrn, dies jedoch im Auftrag

${ }^{41}$ Blumenthal, in: Altorientalische Forschungen IV, 1976 (Anm. 10), S. 35 bzw. S. 37, spricht von „Selbstbewußtsein“ und sogar „Selbstherrlichkeit". des Königshauses (.. ir.t sšm $n n b=i m$ wp.wt $p r(. w)-n z w, Z$ Z. 7, s. schon zuvor). Diese Textstellen machen deutlich, wie sich das Machtgefüge darstellt, dass nämlich der Flottenvorsteher Ntr.w-htp.w für seinen Herrn Nhr.i tätig ist und dieser in der Weisung des Königshauses steht. Auch der im.í-r' $\check{s}(. i) S b k-m-h 3 . t$ äußert sich in einem Graffito aus der Zeit des Nhr.i (,7. Jahr") in dem Sinn, dass er geschickt wurde, um für ihn, nämlich Nhr.i, Alabaster für das Königshaus zu holen $(\ldots r$ in.t $n=f$ ' $n h . w n$ pr(.w)-nzw, Z. 14$)^{42}$.

Letztlich spricht auch schon R. Anthes für Nhr.i von dessen ,oft betonte(m) Ausdruck seiner Ergebenheit dem Könige gegenüber ${ }^{\text {‘3 }}$. Neben den Tätigkeiten im Dienste des Königshauses führt er dafür auch Titel an, die eine Beziehung zum Königshaus aufweisen, sowie eulogische Prädikate, die das gute Auskommen bezeugen. Angesichts seiner übrigen Argumentation erhielten diese Beobachtungen aber nicht die notwendige Berücksichtigung. Die Akzep$\operatorname{tanz}$ des Königshauses als oberste Instanz im Land, so wie sie in den Texten zum Ausdruck gebracht wird, macht aber eine Nennung der eigenen Amtsjahre an Stelle einer königlichen Datierung unannehmbar ${ }^{44}$. In diese Sichtweise fügt sich auch die Angabe von 'Imn.i im benachbarten 16. oberägyptischen Nomos ein, der in der biographischen Inschrift seines Grabes das 43. Jahr von Sesostris I. als sein 25. Amtsjahr bezeichnete, also gleichfalls die Anbindung an die königlichen Regierungsjahre suchte ${ }^{45}$.

(2) In den meisten Graffiti folgen Jahresangabe und Name des Nomarchen direkt hintereinander. Es ließe sich also in der Tat ,Jahr X

${ }^{42}$ Weniger eindeutig ist Gr. 20 aus der Zeit des Nhr.i (,6. Jahr ${ }^{6}$ ) mit der Formulierung $\underline{d} d(. w) n n z w, w d=f$ (i) $r=f$, iwj hrw.w $n(. i) n d w . t-r$ ' ,der zum König spricht, dass er ihm befehlen möge, wenn der Tag der Beratung kommt" (Z. 4f.).

${ }^{43}$ Anthes, Hatnub (Anm. 4), S. 91.

44 Ähnlich bereits Brovarski, in: Fs Dunham (Anm. 13), S. 27, der allerdings nur für die 12. Dynastie (wie auch für das Alte Reich) eine Übernahme königlicher Privilegien und eine Datierung der Nomarchen nach eigenen Amtsjahren für unwahrscheinlich hält.

${ }^{45}$ P. E. Newberry, Beni Hasan I, ASE 1, London 1893, Tf. VIII; Urk. VII, S. 14, 3 ff. 
des (Nomarchen) Y" lesen ${ }^{46}$. Dem stehen zwei Fälle gegenüber, bei denen auf Grund der Anordnung innerhalb des Textes Jahr und Name getrennt und entsprechend nicht aufeinander zu beziehen sind. Beide Graffiti (Gr. 9 und $11^{47}$ ) gehören zu den frühesten der hier besprochenen, wobei die zeitliche Einordnung von Gr. 9 des ' $T h 3$ unsicher ist (s. bereits zuvor). Bei diesem Graffito ist die Jahresangabe „31. Jahr, zweiter Monat der 3h.t-Jahreszeit" sicher eine Datierung, die auf eine königliche Regierungszeit zu beziehen ist. Sie ist in einer (waagerechten) Zeile unter den Haupttext gesetzt, Titel und Name des Ih3 stehen in einer (senkrechten) Kolumne davor. In Gr. 11, das nun sicher aus dem frühen Mittleren Reich stammt, steht die Angabe „30. Jahr" waagerecht über dem Haupttext, Titel und Name des Nomarchen, letzterer allerdings zerstört, stehen senkrecht davor. Es besteht also keinerlei Notwendigkeit, Jahreszahl und Nomarch aufeinander zu beziehen, die Anordnung spricht sogar deutlich dagegen.

Zudem ist auf eine Formulierung in Gr. 21 hinzuweisen, die eine Notiz des Schreibers von Gr. 20 enthält ${ }^{48}$. Es heißt darin, dass die Stele für Nhr.i (I.) im 6. Jahr angefertigt wurde: (1) in []

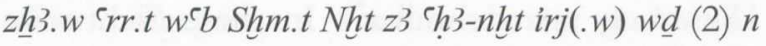
h3.ti-`.w hrp ns.ti im.i-r' hm.w-ntr Nhr.i 'nh wd snb $m$ rnp.t 6 rnp.t () (3) ... ${ }^{49}$. Das 6. Jahr ist auch zu Beginn von Gr. 20 genannt, doch gibt die Formulierung in Gr. 21 kein Indiz dafür, dass tatsächlich die Amtszeit des Nhr.i gemeint ist.

Die genannten Indizien reichen m. E. aus, in allen Graffiti Jahresangabe und Name des Nomarchen zu trennen, in der Jahresangabe die königliche Datierung zu erkennen und in der Namensnennung des Nomarchen die jeweilige

${ }^{46}$ Dies betrifft Gr. 10, 12, 14-16, 19/20, 22, 25/26, 28 und 31 .

${ }^{47}$ Vgl. zum Folgenden Anthes, Hatnub (Anm. 4), S. 23f. und Tfn. 13/13a (Gr. 9) sowie S. 32f. und Tf. 14 (Gr. 11).

${ }^{48}$ S. op. cit., S. 47 und Tf. 20.

49 Auf das zweite rnp.t folgt vermutlich eine Formulierung, die das Jahr mit einem bestimmten Ereignis in Verbindung bringt. Die Stelle ist allerdings so zerstört, dass sich Genaueres nicht sagen lässt, vgl. dazu op. cit., S. 47. regionale Zuständigkeit. Diese Annahme schließt nicht aus, dass mit diesen beiden Elementen bei der Niederschrift der Texte in gewisser Weise gespielt wurde. Für $N h r . i$ (I.) ist zu überlegen, ob sein Amtsantritt mit dem königlichen Herrschaftsbeginn zusammenfiel, beide also das gleiche Jahr zählten. Bei dem früheren `h3-nht (I.) mögen die historischen Verhältnisse für die Auslassung oder Unterdrückung des Königsnamens mit entscheidend gewesen sein (zu beidem s. noch im Folgenden).

Dessen ungeachtet wird man davon ausgehen können, dass die Nomarchen von Hermopolis innerhalb ihrer Beamtengruppe eine besondere Stellung innehatten. Bei Nhr.i (I.) und seinen Söhnen wird dies mit dem Einsatz und den Leistungen der Familie bei den Unruhen zum Ende der 11. Dynastie und der Loyalität gegenüber dem neuen Herrscher Amenemhet I. zusammenhängen. Es ist aber auch auffällig, dass schon in der 11. Dynastie unter Mentuhotep II. mit ' $h 3$-nht (I.) und seinen beiden Söhnen eine Nomarchenfamilie in Hermopolis eingesetzt worden ist, da ansonsten die Reform dieser Verwaltungsstruktur nicht vorangetrieben wurde ${ }^{50}$, anders als es die Politik Amenemhets I. verfolgte. Hierfür wird man andere Gründe suchen müssen, die z. B. im religionspolitischen Bereich liegen könnten ${ }^{51}$.

\section{Die absolute Datierung der Nomarchen}

Löst man sich von der Vorstellung, dass sich die Nomarchen von Hermopolis in den Graffiti von Hatnūb als Inhaber von Regierungs- bzw. Amtsjahren gerieren, so ergibt sich daraus eine neue Möglichkeit für die (absolute) Datierung

${ }^{50}$ L. Gestermann, Kontinuität und Wandel in Politik und Verwaltung des frühen Mittleren Reiches in Ägypten, GOF IV/18, Göttingen 1987, S. 138 ff., S. 171 und passim.

Ead., Sargtexte aus Dair al-Biršā. Zeugnisse eines historischen Wendepunktes?, in: S. Bickel/B. Mathieu (Hrg.), D'un monde à l'autre. Textes des Pyramides \& Textes des Sarcophages. Actes de la table ronde internationale „Textes des Pyramides versus Textes des Sarcophages“. IFAO - 24-26 septembre 2001, BdE 139, Le Caire 2004, S. 201-217. 
dieser Texte. Neben den Datumsangaben in den Inschriften ist es - in einem ersten Schritt - die relative Chronologie der Nomarchen, die mit heranzuziehen ist (s. zuvor), denn die Jahresangaben, die vom Namen eines Nomarchen gefolgt werden, finden sich in den Graffiti von Hatnūb bei insgesamt drei (eventuell vier) Männern, deren relative zeitliche Stellung zueinander gesichert ist. In den Graffiti sind insgesamt 10 Regierungsjahre genannt, die auf drei Herrscher zu verteilen sind: Das 13. (Gr. 12) und 20. Jahr (Gr. 10) eines Pharao, unter dem $\$$ h3-nht (I.) amtierte, das 30. Jahr (Gr. 11) desselben Königs, entweder aus der Amtszeit von ${ }^{(h 3}$ - $n h$ ht (I.) oder der einer seiner Söhne, das 4. (Gr. 14/15), 5. (Gr. 16-19), 6. (Gr. 20/21), 7. (Gr. 22-25) und das 8. Jahr (Gr. 26-28) eines Herrschers, für den Nhr.i (I.) tätig war. Nhr.i (I.) folgte auf 'h3-nht (I.) und dessen Sohn bzw. dessen Söhne. Und schließlich wird ein 20. (Gr. 32) und ein 2. Jahr (Gr. 31) in den Graffiti von und für Dhw.ti-nht (IV.), Sohn von Nhr.i (I.) erwähnt. Die beiden Jahresnennungen zeigen offensichtlich einen Herrscherwechsel an.

Damit ist eine Sequenz von Regierungsjahren verfügbar, die in die Königsabfolge eingehängt werden kann, allerdings ergeben sich auch aus dieser Reihung zunächst einmal zwei Möglichkeiten einer absoluten Datierung. Zum einen lassen sich die erwähnten Regierungsjahre auf die Pharaonen Mentuhotep II. (13., 20. und 30. Jahr), Amenemhet I. (4.-8. und 20. Jahr) und Sesostris I. (2. Jahr) verteilen, zum anderen auf Amenemhet I. (13., 20. und 30. Jahr), Sesostris I. (4.-8. und 20. Jahr) und Amenemhet II. (2. Jahr). Unter Hinzunahme weiterer Faktoren und Überlegungen lassen sich die beiden Möglichkeiten aber gegeneinander abwägen, und es lässt sich, wie ich meine, der zeitlich spätere Ansatz weitgehend, wenngleich vielleicht nicht mit letzter Sicherheit, ausschließen.

Bei der absoluten Datierung der Nomarchen ist zunächst zu berücksichtigen, dass im Lauf der Regierungszeit von Sesostris I. eine Veränderung bei der Datierung der Graffiti zu beobachten ist. Es wird nun nämlich eine andere, neue Art der Datierung eingeführt, bei der das angegebene Jahr (sofern vorhanden) mit dem Namen eines Herrschers verbunden ist. Die
Nennung von Nomarchen wird demgegenüber zurückgedrängt, das Graffito von $\underline{D} h w . t i-n h t$ (V.) („2. Jahr") ist das letzte Beispiel aus Hatnūb, bei dem die Datierung in einem Graffito mit der Nennung eines Nomarchen einhergeht. Im 22. Jahr von Sesostris I. hinterließ der Schatzmeister Sbk-ḥtp eine Stele in Hatnūb, erwähnt darin aber keinen (amtierenden) Nomarchen, da diese Unternehmung offensichtlich im direkten Auftrag des Königshauses durchgeführt wurde ${ }^{52}$. Aus dem 31. Jahr kennen wir ein Graffito des (späteren) Nomarchen 'Imn.w-m-ḩ3.t (Gr. 49). Ohne Jahresangabe blieb eine weitere Inschrift von einem Truppenführer, die im Datum nur Sesostris I. nennt ${ }^{53}$. Aus dem kleinen Steinbruch schließlich stammt ein Graffito, das im 20. Jahr Amenemhets II. dort angebracht wurde (Gr. 50). Es ist also offensichtlich in der ersten Hälfte der Regierungszeit von Sesostris I. eine Art der Datierung (ohne Königsnennung, aber mit dem Namen des Nomarchen) durch eine andere (allein mit Königsnennung) abgelöst worden.

Wählt man nun den späteren der beiden Datierungsansätze (Amenemhet I. - Amenemhet II.), so hätte dies für die ersten Nomarchen ' ḩ3-nht (I.) und Sohn/Söhne sowie Nhr.i (I.) das bekannte Für und Wider zur Folge. Es ließen sich - vor allem mit Hinweis auf Paläographie und Inhalte der entsprechenden Texte - sowohl für die Datierung von ' $h 3$ - nht (I.) in die Regierungszeit Amenemhets I. und die von Nhr.i (I.) in die Zeit von Sesostris I. als auch gegen diese zeitliche Einordnung Argumente anführen. Für Dhw.ti-nht (V.) aber sind Konsequenzen aufzeigen, die zu internen Widersprüchen führen. Bei einer späteren Datierung hätte Dhw.ti-nht (V.) mindestens vom 20. Jahr Sesostris' I. bis zum 2. Jahr von Amenemhet II. amtiert, d. h. wenigs-

${ }^{52}$ B. Grdseloff, Un noveau graffito de Hatnoub, in: ASAE LI, 1951, S. 143-146, mit dem Fragment einer Inschrift, das sich an ein zweites, größeres Stück anpassen lässt. Beide zusammen wurden von $G$. Posener, Une Stèle de Hatnoub, in: JEA 54, 1968, S. 67-70 und Tfn. VIIIf, veröffentlicht.

W. K. Simpson, A Hatnub Stela of the Early twelfth Dynasty, in: MDAIK 16, 1958, S. 298-309 und Tfn. XXIXf., mit einem zusätzlichen Fragment id., An Additional Fragment of a „Hatnub“ Stela, in: JNES XX, 1961, S. 25-30, publiziert. 
tens 26 Jahre, was nachvollziehbar erscheint. Seine Tätigkeit als Nomarch würde dann allerdings in eine Zeit fallen, in der bereits (wieder) Königsnennungen - allein oder mit Jahresangabe - in die Inschriften der Nomarchen aufgenommen wurden. Dies steht in einem gewissen Widerspruch zu Gr.31, in dem der Name von Dhw.ti-nht (V.) direkt hinter der Angabe „2. Jahr" steht, also noch die älteren Gepflogenheiten geübt werden. Schwerer wiegt allerdings, dass wir aus der Zeit von Sesostris I. Imn.w-m-ḩ.t kennen, Sohn von Nhr.i (II.), der mit Gr. 49 aus dem 31. Jahr von Sesostris I. einen Fixpunkt setzt. Bevor Imn.w-m-h3.t überhaupt in dieser Weise handlungsfähig und somit erwachsen ist, hatte möglicherweise sein Bruder Dhw.ti-nht (VI.), hatte auf jeden Fall aber sein Vater Nhr.i (II.) das Nomarchenamt inne. Dies kollidiert jedoch mit einer angenommenen $\mathrm{Da}$ tierung von Dhw.ti-nht $(\mathrm{V}$.) in diese Zeit.

Vor diesem Hintergrund bleibt als einzige Möglichkeit, den früheren zeitlichen Ansatz für eine Datierung von $\$ h 3-n h t$ (I.) und $N h r . i$ (I.) zu wählen und sie den Regierungszeiten von Mentuhotep II. bzw. Amenemhet I. zuzuordnen. In der Folge reichen die zur Diskussion stehenden Graffiti von Mentuhotep II. bis in den Beginn der Herrschaft von Sesostris I. (dazu die beiden folgenden Tabellen) ${ }^{54}$.

Als frühester Nomarch des Mittleren Reiches ist ' $h 3-n h t$ (I.) in die Regierungszeit von Mentuhotep II. zu datieren ${ }^{55}$. Aus dessen 13. und 20. Jahr stammen die beiden Graffiti, die ' $h 3-n h t$ als amtierenden Nomarchen nennen. Gr. 11 aus

\footnotetext{
${ }^{54}$ Allenfalls eines ist (auch) in diesem Fall zu überdenken, und das ist die zeitliche Einordnung von $T h$, der Gr. 9 hinterlassen hat (s. zuvor mit Anm. 37). Je nachdem, wie man sich der zeitlichen Einordnung von Ih3 aus Gr. 9 mit der Datierung in das 31. Jahr eines nicht genannten Pharao gegenüber verhält, ist auch dieser für die Abfolge der Nomarchen am Ende der 11. Dynastie noch zu berücksichtigen. Zurzeit spricht die Nähe von Gr. 9 zu denen des Alten Reiches zwar gegen eine so späte Einfügung des Graffitos, die Bemerkung von E. Brovarski (s. Anm. 37) relativiert dies aber.

${ }^{55}$ Willems, in: JEOL 28, 1983-1984 (Anm. 15), S. 102, Anm. 153, schlägt eine Datierung in die drei letzten Dekaden der 11. Dynastie vor, wählte also einen etwas späteren Ansatz.
}

dem 30. Jahr Mentuhoteps II. wurde entweder gleichfalls noch in der Amtszeit von ' $h 3-n h t$ angebracht oder in der eines der beiden Söhne Th3-nht (II.) und Dhww.ti-nht (IV.) ${ }^{56}$. Die Datierung nach den Regierungsjahren von Mentuhotep II. so weit im Norden des Landes kann nur als Indiz dafür angesehen werden, dass bereits in dessen 13. Jahr die (neuerliche) Reichseinigung erfolgt war. In die gleiche Richtung deutet auch das Grab von 'ḥ3-nht in Dair al-biršā (Grab 5) ${ }^{57}$, und zwar in zweierlei Hinsicht. Zum einen setzen Formulierungen in der biographischen Inschrift auf der Grabfassade voraus, dass sie zeitlich nach der Reichseinigung entstanden $\operatorname{sind}^{58}$, zum anderen weist das Grab die Zusammenführung zweier unterschiedlicher Stilrichtungen auf. Wie R. E. Freed dargelegt hat ${ }^{59}$, wurde die innere Kammer des Grabes nach oberägyptischem Kanon vor der Reichseinigung unter Mentuhotep II. gestaltet, die äußere Kammer in Stil und Ikonographie des (memphitischen) Kanons des Alten Reiches. Dieses Indiz lässt sich ergänzend neben die einleitenden Bemerkungen zur absoluten Datierung von ‘ h3-nht (I.) stellen.

Die fehlende Königsnennung kann man auf die unsichere politische Lage zurückführen: Das Machtgefüge der Thebaner ist bis weit in den Norden ausgedehnt worden, mit einiger Wahrscheinlichkeit dürfte der herakleopolitanischen Seite auch die endgültige Niederlage um die Vorherrschaft in Ägypten zugefügt worden sein, man wird aber gleichzeitig noch nicht von einer stabilen Lage im Land gesprochen haben können. Die abgeänderte Inschrift in den Gräbern von Asyūt zeigt deutlich, wie schnell sich die politischen Verhältnisse drehen konnten und dass man darauf reagiert hat.

\footnotetext{
${ }^{56}$ Ein weiterer Sohn, der Schreiber Hnm.w-iqr (Gr. 10), hat das Nomarchenamt nie innegehabt.

Griffith/Newberry, El Bersheh II (Anm. 5), S. 30-35 und Tfn. XII-XVII; Brovarski, in: Fs Dunham (Anm. 13), S. 14-21; Bersheh Reports I (Anm. 18), S. $43 \mathrm{ff}$. und S. $53 \mathrm{ff}$.

So bereits Brovarski, in: Fs Dunham (Anm. 13), S. 21; s. a. Willems, in: JEOL 28, 1983-1984 (Anm. 15), S. 102, Anm. 153.

Bersheh Reports I (Anm. 18), S. 53ff.
} 
Tabelle 1: Die Nomarchen in den Regierungszeiten von Mentuhotep II., III. und IV.

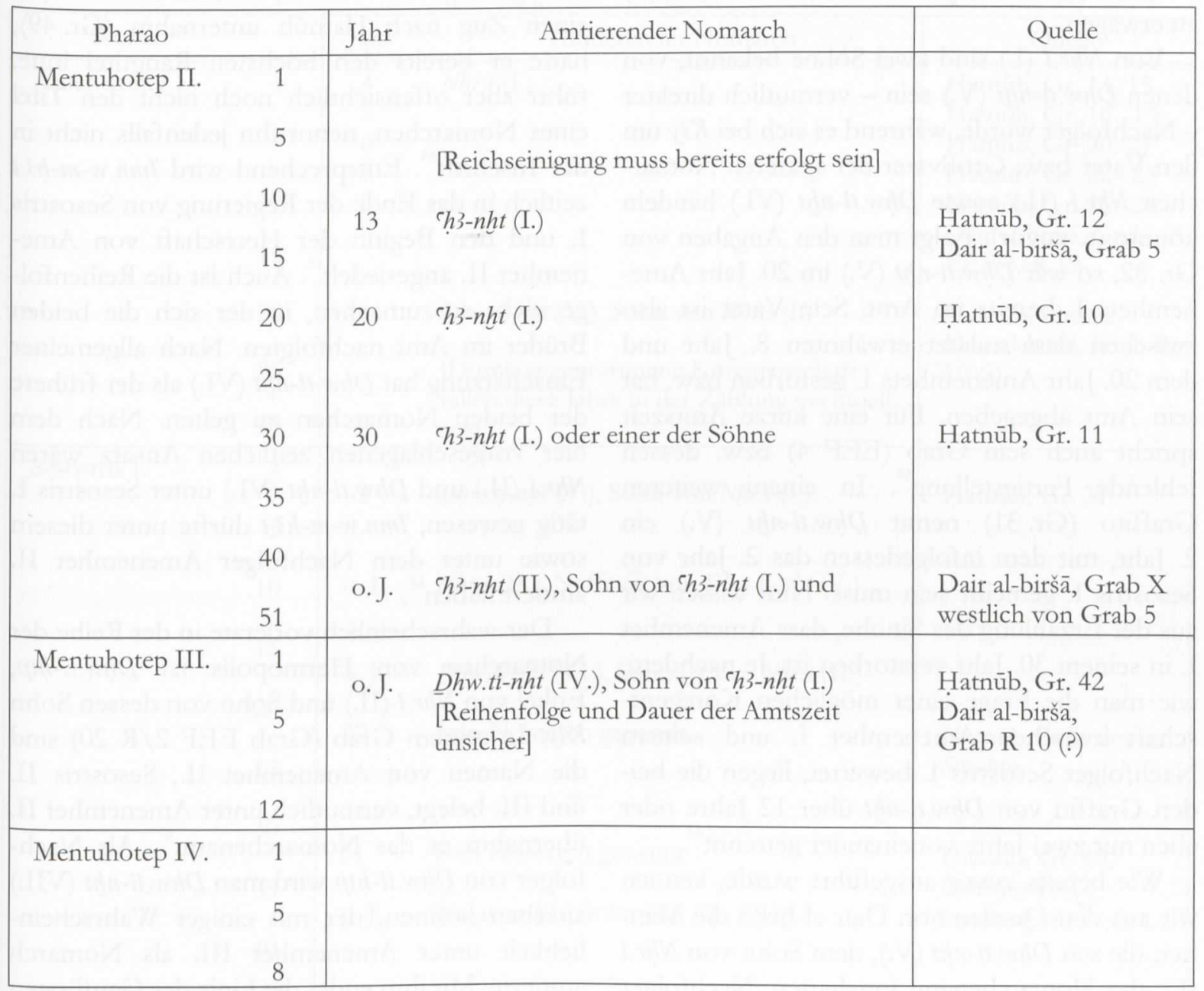

Aus den letzten 20 Regierungsjahren von Mentuhotep II. sowie aus der Zeit von Mentuhotep III. und IV. sind in Hatnūb keine Nennungen von Nomarchen bekannt, die mit einer Jahresangabe einhergingen. Zumindest teilweise wird man diese Epoche mit den Söhnen von `h3-nht (I.) füllen können, sicher mit Dhw.ti-nht (IV.), von dem sich ein Graffito erhalten hat (Gr. 42). Es ist zudem wahrscheinlich, dass ' h3-nht (II.) ebenfalls als Nomarch amtierte. Zum Ende der 11. Dynastie wird die Situation möglicherweise auch so gewesen sein, dass keine klaren Zuständigkeiten mehr bestanden. $\mathrm{Zu}$ bemerken ist jedenfalls, dass sich die Nachkommen von ' $h 3-n h t$ (I.) in ihrem Nomarchenamt nicht in einer Weise profilieren konnten, dass ihnen auch noch in der 12. Dynastie diese Funktion erhalten blieb - aus welchen Gründen auch immer.
Mit ihren Datierungen in das 4. bis 8. Jahr gehören die Graffiti aus der Amtszeit des Nomarchen Nhr.i (I.) in den Beginn der Regierung Amenemhets I. ${ }^{60}$. Mit der 12. Dynastie wird in Hermopolis also eine neue Nomarchenfamilie installiert. Mit einiger Wahrscheinlichkeit wird diese Einsetzung wegen der besonderen Rolle der Familie bei der Überwindung der innenpolitischen Schwierigkeiten zum Ende der 11. Dynastie und ihrer Verdienste um Königshaus und Land erfolgt sein ${ }^{61}$. Es ist deshalb auch ein gleichzeitiger Beginn der Regierungszeit von

${ }^{60}$ Vgl. Willems, in: JEOL 28, 1983-1984 (Anm. 15), S. 94 ff. und S. $101 \mathrm{f}$, mit einer Datierung für Nhr.i in das Ende der 11. oder den Beginn der 12. Dynastie, s. a. id., Chests of Life (Anm. 16), S. 70.

${ }^{61} \mathrm{Zu}$ den Beschreibungen der Unruhen vgl. id., in: JEOL 28, 1983-1984 (Anm. 15), S. 95 ff. 
Amenemhet I. und der Amtszeit von Nhr.i (I.) zu erwägen.

Von Nhr.i (I.) sind zwei Söhne bekannt, von denen Dhw.ti-nht (V.) sein - vermutlich direkter - Nachfolger wurde, während es sich bei $K 3 y$ um den Vater bzw. Großvater der späteren Nomarchen Nhr.i (II.) sowie Dḥw.ti-nht (VI.) handeln könnte (s. zuvor). Folgt man den Angaben von Gr. 32, so war Dhw.ti-nht (V.) im 20. Jahr Amenemhets I. bereits im Amt. Sein Vater ist also zwischen dem zuletzt erwähnten 8. Jahr und dem 20. Jahr Amenemhets I. gestorben bzw. hat sein Amt abgegeben. Für eine kurze Amtszeit spricht auch sein Grab (EEF 4) bzw. dessen fehlende Fertigstellung ${ }^{62}$. In einem weiteren Graffito (Gr. 31) nennt Dhw.ti-nht (V.) ein 2. Jahr, mit dem infolgedessen das 2. Jahr von Sesostris I. gemeint sein muss. Nun wissen wir aus der Erzählung des Sinuhe, dass Amenemhet I. in seinem 30. Jahr verstorben ist. Je nachdem, wie man die Frage einer möglichen Koregentschaft zwischen Amenemhet I. und seinem Nachfolger Sesostris I. bewertet, liegen die beiden Graffiti von Dhw.ti-nht über 12 Jahre oder eben nur zwei Jahre voneinander getrennt ${ }^{63}$.

Wie bereits zuvor ausgeführt wurde, kennen wir aus den Quellen von Dair al-biršā die Männer, die seit $\underline{D} h w . t i-n h t$ (V.), dem Sohn von Nhr.i (I.), das Nomarchenamt innehatten. Nachfolger von Dhw.ti-nht (V.) wurde zunächst $N h r . i$ (II.), vermutlich der Neffe von $D h w . t i-n h t$, Sohn von dessen Bruder K3y. Danach werden zwei Männer in das Nomarchenamt eingesetzt, die vermutlich beide als Söhne von Nhr.i (II.) zu gelten haben. Dies sind Dhw.ti-nht (VI.) sowie Imn.w-m- $h 3 . t$ aus Gr. 49. Für diese beiden ist auf Grund der Datierung von Gr. 31 des Nomarchen $\underline{D} h w . t i-n h t(\mathrm{~V}$.$) in das 2. Jahr von Sesostris$ I. nachweisbar, dass sie nach diesem Zeitpunkt in das Amt eingesetzt wurden. Ein genauerer Zeitpunkt lässt sich aber nicht bestimmen - im

${ }^{62}$ Zu Grab EEF 4/R 19 Griffith/Newberry, El Bersheh II (Anm. 5), S. 29 und Tfn. Xf.; Bersheh Reports I (Anm. 18), S. 29 f., S. $61 \mathrm{ff.} \mathrm{zu} \mathrm{der} \mathrm{Datierung} \mathrm{in}$ die frühe 12. Dynastie und den Parallelen.

${ }^{63}$ Willems, Chests of Life (Anm. 16), S. $70 \mathrm{ff}$., kommt für Dhw.ti-nht (V.) auf eine Datierung in das Ende der Regierungszeit von Amenemhet (I.).
31. Jahr von Sesostris I., in dem Imn.w-m-h3.t einen Zug nach Hatnūb unternahm (Gr. 49), hatte er bereits den höchsten Rangtitel inne, führt aber offensichtlich noch nicht den Titel eines Nomarchen, nennt ihn jedenfalls nicht in der Inschrift ${ }^{64}$. Entsprechend wird Imn.w-m- $h 3 . t$ zeitlich in das Ende der Regierung von Sesostris I. und den Beginn der Herrschaft von Amenemhet II. angesiedelt ${ }^{65}$. Auch ist die Reihenfolge nicht auszumachen, in der sich die beiden Brüder im Amt nachfolgten. Nach allgemeiner Einschätzung hat Dhww.ti-nht (VI.) als der frühere der beiden Nomarchen zu gelten. Nach dem hier vorgeschlagenen zeitlichen Ansatz wären Nhr.i (II.) und Dhw.ti-nht (VI.) unter Sesostris I. tätig gewesen, Imn.w-m-h3.t dürfte unter diesem sowie unter dem Nachfolger Amenemhet II. amtiert haben ${ }^{66}$.

Der wahrscheinlich vorletzte in der Reihe der Nomarchen von Hermopolis ist Dhw.ti-htp, Enkel von Nhr.i (II.) und Sohn von dessen Sohn K3y. In seinem Grab (Grab EEF 2/R 20) sind die Namen von Amenemhet II., Sesostris II. und III. belegt, vermutlich unter Amenemhet II. übernahm er das Nomarchenamt ${ }^{67}$. Als Nachfolger von $\underline{D} h w . t i-h t p$ wird man Dh $h w . t i-n h t$ (VII.) ansehen können, der mit einiger Wahrscheinlichkeit unter Amenemhet III. als Nomarch amtierte. Mit ihm endet die Linie der Gaufürsten im 15. oberägyptischen Nomos, die immerhin von sich sagen können, das Amt vom Beginn der 12. Dynastie bis zumindest unter Sesostris III. in einer Familie gehalten zu haben.

${ }^{64}$ S. a. Willems, in: JEOL 28, 1983-1984 (Anm. 15), S. 84, der allerdings von einem niedrigeren Rangtitel ausgeht.

${ }_{65}$ Bersheh Reports I (Anm. 18), S. 28; Willems, Chests of Life (Anm. 16), S. 74 f.

${ }^{66}$ Willems, op. cit., S. 74, schlägt für das Grab von Nhr.i (II.) (B1B) eine Datierung in die zweite Hälfte der Regierung von Sesostris I. vor. Die beiden Söhne gehören nach seiner Einschätzung, op. cit., S. 75, gleichfalls in die Zeit von Sesostris I. und den Beginn der Regierung Amenemhets II.; s. a. Brovarski, in: Fs Dunham (Anm. 13), S. $23 \mathrm{ff}$.

${ }^{7} \mathrm{Zu}$ diesem Nomarchen und seinen Quellen, vor allem Grab 2 in Dair al-biršā, vgl. Willems, Chests of Life (Anm. 16), S. 75 ff.; Brovarski, in: Fs Dunham (Anm. 13), S. 25. 
Tabelle 2: Die Nomarchen in den Regierungszeiten von Amenemhet I., Sesostris I. und Amenemhet II.

\begin{tabular}{|c|c|c|c|c|}
\hline Pharao & & Jahr & Amtierender Nomarch & Quelle \\
\hline Amenemhet I. & $\begin{array}{l}15 \\
20\end{array}$ & $\begin{array}{l}4 \\
5 \\
6 \\
7 \\
8 \\
\end{array}$ & $\begin{array}{l}\text { Dhw.ti-nht (V.), Sohn von Nhr.i (I.) } \\
\text { [Durch angenommene Koregentschaft } \\
\text { fallen diese Jahre in der Zählung eventuell } \\
\text { aus] }\end{array}$ & $\begin{array}{l}\text { Hatnūb, Gr. 14/15 } \\
\text { Hatnūb, Gr. 16-19 } \\
\text { Ḧatnūb, Gr. 20/21 } \\
\text { Hatnūb, Gr. 22-25 } \\
\text { Hatnūb, Gr. 26-28 } \\
\text { Dair al-biršā, Grab } \\
\text { EEF 4/R 19 } \\
\text { Hatnūb, Gr. } 32 \\
\text { Dair al-biršā, Grab R } \\
10 \text { (?) }\end{array}$ \\
\hline Sesostris I. & $\begin{array}{l}35 \\
40 \\
44\end{array}$ & $\begin{array}{l}2 \\
\text { o. J. } \\
\text { o. J. } \\
22 \\
31 \\
\text { o. J. }\end{array}$ & 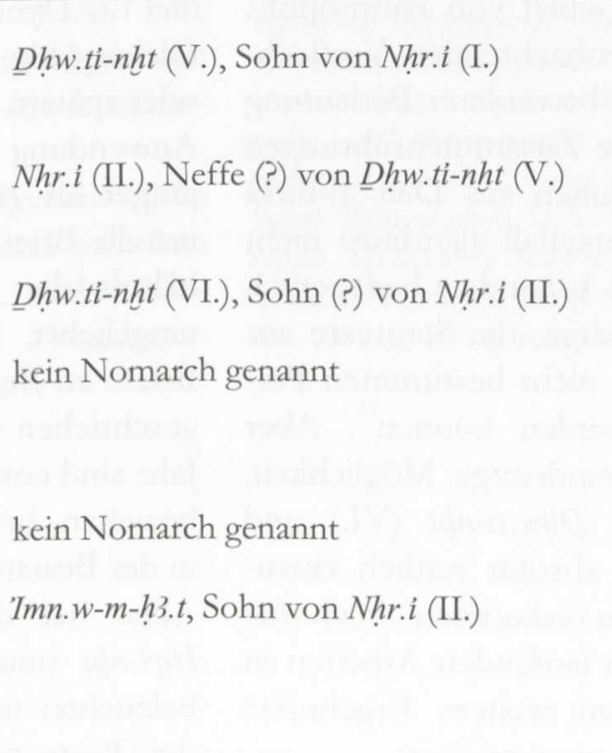 & $\begin{array}{l}\text { Hatnūb, Stele des } \\
\text { Sbk-h.htp } \\
\text { Hatnūb, Gr. } 49 \text {, } \\
\text { Graffito von } \\
\text { Imn.w-m- } h 3 . t\end{array}$ \\
\hline Amenemhet II. & 10 & & $\begin{array}{l}\text { Dhw.ti-htp } \\
\text { [amtierte bis unter Sesostris III.] }\end{array}$ & $\begin{array}{l}\text { Dair al-biršā, Grab } \\
\text { EEF 2/R } 20\end{array}$ \\
\hline
\end{tabular}

\section{Das zeitliche Umfeld}

Kehren wir noch einmal zu der Annahme zurück, dass die Jahresangaben in den früheren Inschriften das aktuelle Regierungsjahr des herrschenden Königs meinen. Diese Interpretation, für die es m. E. gute Gründe gibt, birgt einen gänzlich neuen Ansatz, die Datumsangaben in den Graffiti in das zeitliche Gerüst einzufügen, das uns die Herrscherabfolge des frühen Mittleren Reiches vorgibt. Zudem sind nun wesentlich genauere Datierungen möglich.
Wie ich zuvor gleichfalls ausgeführt habe, möchte ich die Jahresangaben in den Graffiti so zuordnen, dass für (h3-nht (I.) eine Amtszeit unter Mentuhotep II. und für Nhr.i (I.) eine Amtszeit unter Amenemhet I. anzunehmen ist. Dafür bzw. gegen den von W. Schenkel vorgeschlagenen späteren Ansatz Amenemhet I./ Sesostris I., für den er vornehmlich aufgrund seiner Ablehnung einer Datierung der beschriebenen Unruhen in die Erste Zwischenzeit argumentiert hatte, sprechen vor allem genealogische Überlegungen, die sich an bestimmten zeitlichen 
Fixpunkten zu orientieren haben, daneben aber auch Hinweise aus anderen Quellen. Diese absolute Datierung stimmt im Wesentlichen mit dem Ansatz von $\mathrm{H}$. Willems überein, doch ergeben sich einige kleinere Verschiebungen. Letztlich möchte ich allerdings nicht vollständig ausschließen, dass sich in der Frage der absoluten zeitlichen Einordnung noch Veränderungen ergeben könnten. Hier gilt es, die weiteren Ergebnisse der archäologischen Unternehmung in Dair al-biršā abzuwarten.

Die Implikationen, die sich aus einer absoluten Datierung der Graffiti von Hatnūb ergeben (können), sind für das Gebiet von Hermopolis aufgrund der dort zu beobachtenden Verflechtungen der Quellen von besonderer Bedeutung (s. bereits einleitend). Die Zusammenführungen zwischen den Informationen aus Dair al-biršā und Hatnūb sind im Einzelfall allerdings nicht ohne Probleme, wobei es besonders bedauerlich ist, dass die frühesten Särge, die Sargtexte aus Dair al-biršā überliefern, nicht bestimmten Personen zugeschrieben werden können ${ }^{68}$. Aber auch die bislang nicht eindeutige Möglichkeit, die beiden Nomarchen Dhw.ti-nht (VI.) und Imn.w-m-h3.t relativ wie absolut zeitlich einzuordnen, ist noch weiter zu diskutieren. Auch hier ist auf die augenblicklich laufenden Arbeiten in Dair al-biršā, von denen weitere Ergebnisse erwartet werden können, zu verweisen.

Zuvor sind die Quellen aus Hatnūb und Dair al-biršā als eigene Textkonvolute und isoliert vom sonstigen historischen Hintergrund betrachtet worden. Damit ist nicht die inhaltliche Bezugnahme auf die Ereignisse der Zeit gemeint, die ja zur Sprache kamen, sondern das zeitliche Verhältnis, das zwischen diesen Texten und anderen aus etwa der gleichen Epoche besteht. Im Fall der Graffiti von Hatnūb können das z. B. die Reisner-Papyri, Expeditionsinschriften von anderen Unternehmungen oder die Briefe des $H q 3$ - $n h t$ sein, die insgesamt vergleichbare Charakteristika besitzen. Um die Relevanz einer solchen Betrachtungsweise deutlich zu machen, seien die Briefe des $H q 3$ - $n h t$ heraus-

\footnotetext{
${ }^{68}$ Brovarski, in: Fs Dunham (Anm. 13), S. $14 \mathrm{ff}$., Willems, in: JEOL 28, 1983-1984 (Anm. 15), S. 80 ff., id., Chests of Life (Anm. 16), S. $70 \mathrm{ff.}$
}

gegriffen, die erst kürzlich eine Neubearbeitung erfahren haben ${ }^{69}$.

Die zeitliche Einordnung der Graffiti von Nhr.i (I.) und der Briefe des $H q 3$ - nht ist zunächst, d. h. in der frühen Phase der wissenschaftlichen Auseinandersetzung mit ihnen, in etwa gleich gewesen. Bei beiden sollte es sich um Zeugnisse aus der ausgehenden Ersten Zwischenzeit bzw. dem Beginn des Mittleren Reiches handeln, wobei $H q$ 3-nht auf Grund der Nennung des 5. und 8. Regierungsjahres, das auf Mentuhotep II. bezogen wurde, etwas früher als Nhr.i (I.) anzusetzen war ${ }^{70}$. Mit der Umdatierung von Nhr.i (I.) in die 12. Dynastie entzerrte sich das Verhältnis. Gleichgültig, ob der frühere (Amenemhet I.) oder spätere (Sesostris I.) Ansatz für Nhrr.i (I.) in Anwendung kam, war Nhr.i (I.) nun deutlich jünger als $H q 3-n h t$. Die neuerliche Diskussion um die Briefe des Hq3-nht hat das zeitliche Verhältnis der beiden Textgruppen indes genau umgekehrt. Wie J. P. Allen darlegt, sollen die Briefe zu Beginn der Regierung von Sesostris I. geschrieben worden sein, das erwähnte 5 . und 8 . Jahr sind entsprechend auf dessen Herrschaft zu beziehen, bevor sie kurz danach (versehentlich) in der Bestattung des $M s h$ verloren gingen ${ }^{71}$. J. P. Allen hat die Bezugnahmen der Briefe des $H q 3-n h t$ unter verschiedenen Gesichtspunkten beleuchtet und hat dabei auch die Paläographie der Texte mit anderen zeitnahen Textgruppen wie den Reisner-Papyri und den Graffiti von Hatnūb verglichen, ohne dabei auf Widersprüche gestoßen zu sein ${ }^{72}$. Während der Duktus der Texte ihre derzeitige Datierung also zu bestätigen scheint, fügt sich ein weiteres Kriterium, und zwar die Determinierung in hieratisch geschriebenen Texten der 11./12. Dynastie, zumindest nicht zu dem zeitlichen Verhältnis, in dem die Briefe des Hq3-nht und die Graffiti von Nhr.i (I.) nun zueinander stehen. Wie W. Schen-

${ }^{69}$ J. P. Allen, The Heqanakht Papyri, PMMA XXVII, New York 2002.

${ }^{70} \mathrm{Zu}$ den Briefen des $H q$ 3-nht vgl. T. G. H. James, The Hekanakhte Papers and Other Early Middle Kingdom Documents, PMMA XIX, New York 1962, insbes. S. 2 ff., zu den Graffiti in Hatnūb s. zuvor.

Allen, The Heqanakht Papyri (Anm. 69), S. $127 \mathrm{ff}$. und S. $179 \mathrm{ff}$.

${ }^{72}$ S. insbes. op. cit., S. $84 \mathrm{f}$. 
kel in einer Untersuchung u. a. postuliert hat, steigt bei hieratisch geschriebenen Texten die durchschnittliche Zahl der Determinative pro Wort zwischen der 8. und 12. Dynastie an und nimmt die Zahl der determinativlosen Wörter $\mathrm{ab}^{73}$. Danach wären die Graffiti von $N h r . i$ (I.) jünger als die Briefe des Hq3-nht. Aus dem direkten Vergleich ist zudem abzuleiten, dass die beiden Textgruppen nicht aus derselben Zeit stammen können ${ }^{74}$. Mit der Datierung von Nhr.i (I.) in den Beginn der Regierung von Amenemhet I. und der zeitlichen Einordnung von $H q 3$-nht in die frühe Regierungszeit von Sesostris I. ist Letzteres zwar nicht der Fall, doch müssten sie nach diesem Kriterium an sich in umgekehrter zeitlicher Reihenfolge zueinander stehen.

Es soll im Folgenden dieses Ergebnis nicht weiter diskutiert werden. Der Befund erfordert aber ohne Zweifel eine Klärung, wobei verschiedene Erklärungsmodelle und Vorgehens- weisen im Auge zu behalten sind ${ }^{75}$. Abgesehen davon, dass diese Untersuchungen für die hier angesprochene Fragestellung notwendig wären, könnten sie sicher auch in einem größeren Kontext von Interesse sein.

\section{Summary}

The paper argues for a new interpretation of the dates which are given in some graffiti from Hatnūb belonging to the early Middle Kingdom. It is suggested that these dates do not refer to the nomarchship of 'h3-nht (I), Nhr.i (I) and Dhw.ti-nht (IV) but stand for the reign of a king, even though this king is not mentioned by name. This interpretation presents a new chance to date the nomarchs from Hermopolis, and their tombs at Dair al-biršā along with their equipment, more precisely within the reigns from Mentuhotep II to Sesostris I. It also includes some reflections regarding the chronological relationship between the graffiti from Hatnūb and other documents dating from nearly the same period.
${ }^{73}$ W. Schenkel, Zur Redaktions- und Überlieferungsgeschichte des Spruchs 335 A der Sargtexte, in: W. Westendorf (Hrg.), Göttinger Totenbuchstudien. Beiträge zum 17. Kapitel, GOF IV/3, Wiesbaden 1975, S. 37-79 (S. 76ff.).

$$
{ }^{74} \text { Op. cit., S. } 79 .
$$

${ }^{15}$ Dazu zählt (z. B.) die Möglichkeit, dass regional unterschiedliche Gepflogenheiten bei der hieratischen Wiedergabe eines Textes bestanden. Auch ist die Signifikanz des Ergebnisses weiter zu verfolgen, d. h. es sind zusätzliche Quellengruppen in solch eine Untersuchung mit aufzunehmen. 